\title{
GCTTCA as a novel motif for regulating mesocarp-specific expression of the oil palm (Elaeis guineensis Jacq.) stearoyl-ACP desaturase gene
}

\begin{abstract}
Two key fatty acid biosynthetic genes, stearoyl-ACP desaturase (SAD1), and acylcarrier protein (ACP3) in Elaeis guineensis (oil palm) showed high level of expression during the period of oil synthesis in the mesocarp [12-19 weeks after anthesis (w.a.a.)] and kernel (12-15 w.a.a.). Both genes are expressed in spear leaves at much lower levels and the expression increased by 1.5-fold to 2.5-fold following treatments with ethylene and abscisic acid (ABA). Both SAD1 and ACP3 promoters contain phytohormone-responsive, light-responsive, abiotic factors/wounding-responsive, endosperm specificity and fruit maturation/ripening regulatory motifs. The activities of the full length and six 5' deletion fragments of the SAD1 promoter were analyzed in transiently transformed oil palm tissues by quantitative $\beta$-glucuronidase (GUS) fluorometric assay. The highest SAD1 promoter activity was observed in the mesocarp followed by kernel and the least in the leaves. GUS activity in the D3 deletion construct $(-486$ to +108$)$ was the highest, while the D2 $(-535$ to +108$)$ gave the lowest suggesting the presence of negative cis-acting regulatory element(s) in the deleted -535 to -486 (49 bp). It was found that the 49-bp region binds to the nuclear protein extract from mesocarp but not from leaves in electrophoretic mobility shift assay (EMSA). Further fine-tuned analysis of this 49-bp region using truncated DNA led to the identification of GCTTCA as a novel motif in the SAD1 promoter. Interestingly, another known fruit ripening-related motif, LECPLEACS2 (TAAAAT) was found to be required for effective binding of the novel motif to the mesocarp nuclear protein extract.
\end{abstract}

Keyword: Stearoyl-ACP desaturase; Acyl-carrier protein; Oil palm; Fatty acid biosynthesis; Promoter deletion analysis; TAAAAT motif 
